\title{
Heat transfer and pressure drop experimentation inside single minichannels
}

\author{
A. Cavallini, S. Bortolin, D. Del Col, M. Matkovic \& L. Rossetto \\ Dipartimento di Fisica Tecnica, University of Padova, Italy
}

\begin{abstract}
The present authors have designed and built a test apparatus for heat transfer and pressure drop measurements during forced convective single phase flow, condensation and flow boiling in one single minichannel. The performance of the test apparatus for investigation of heat transfer and fluid flow is described in this paper. Some construction issues with important effects on the experimental technique are also reported in the paper. The adiabatic and diabatic two phase pressure drop, the local heat transfer coefficient and the dry-out phenomenon, as well as the single phase heat transfer coefficient measurements, are all discussed herein. The experimental apparatus has turned out to be highly effective in terms of low experimental uncertainty, local measurement capabilities and rather fast data acquisition rate over a wide range of test conditions. Vast, reliable experimental data have a key role in semi-empirical modelling. Indeed, these experimental results, together with data associated to minichannels from different authors, have been used for the development of new heat transfer and pressure drop models able to accurately predict experimental data for low, medium and high pressure fluids.
\end{abstract}

Keywords: heat transfer, pressure drop, minichannels, condensation, flow boiling, dry-out.

\section{Introduction}

In the field of heat transfer mini-, micro- and nanochannels have gained a great deal of attention in engineered systems. A number of advantages associated with minichannels, with an inner diameter in the range of 0.2-3 $\mathrm{mm}$ [1], have pushed their use far ahead of the actual understanding of the microscale process and how the scaling down alters the governing physical processes. First compact heat 
exchangers in the automotive industry, for example, were integrated into vehicles' air-conditioning systems before they were really optimized in terms of fluid charge, material use and compactness. Optimizing the device performances reduces its size, energy requirements, costs and last, but not the least, impact on the environment [2].

Minichannels adopted in heat transfer engineering can be found in a wide variety of applications: from residential air-conditioning to spacecraft thermal control. Compact elements work with reduced refrigerant charges and can usually withstand extremely high system pressures. A small charge reduces the direct greenhouse effect of the systems and promotes the use of flammable or even toxic refrigerants. Furthermore, resistance to high pressure enables one to use natural refrigerants, such as carbon dioxide, in these systems and hence the operation in the supercritical region.

In order to better understand how the scaling-down alters fluid flow, heat transfer and phase change processes, the present authors designed a special test apparatus for investigation within single minichannels (Matkovic [3]).

\section{Short overview of the test apparatus}

With reference to the test rig in fig. 1, subcooled refrigerant from the postcondenser passes the mechanical filter and dehumidifier. It is pumped with a variable speed electric motor gear pump through the Coriolis effect mass flow meter and the throttling valve into either the evaporator or the sub-cooler, depending upon the desired test condition at the inlet of the test section. Here, the temperature and the pressure measurements define the thermodynamic state of the refrigerant.

An important part of the primary loop is the accumulator (PV). It is used to store a certain amount of the test fluid as a liquid. The system pressure in the rig is controlled by varying the volume of the liquid contained in the accumulator. Since the liquid contained in the accumulator after steady state operation has been reached is the redundant amount of the system fluid, its size has been carefully defined. As the accumulator is partly filled with a compressible gas, such as nitrogen, it works also as an expansion vessel. Therefore, this element should never be detached from the system.

Another important element of the primary refrigerant loop is the throttling valve (TV2). It is installed after the pump, accumulator and Corriolis effect mass flow meter. Its purpose is to make the fluid flow more stable with proper throttling.

The "reset" bypass (dashed line) allows one to send some fluid back to the inlet of the postcondenser. In this way, the pump works at rather high flow rates, and the part of the test rig from the postcondenser down to the outlet of the pump is sufficiently subcooled to avoid possible cavitation. As a consequence, the flow is more stable. The cavitation is likely to occur at low flow rates for the small thermal capacity, and could cause insufficient feeding of the pump and lack of fluid flow through the test section. It is therefore important to have sufficient reflux of the refrigerant, particularly when the saturation temperature is relatively close to the ambient temperature. 


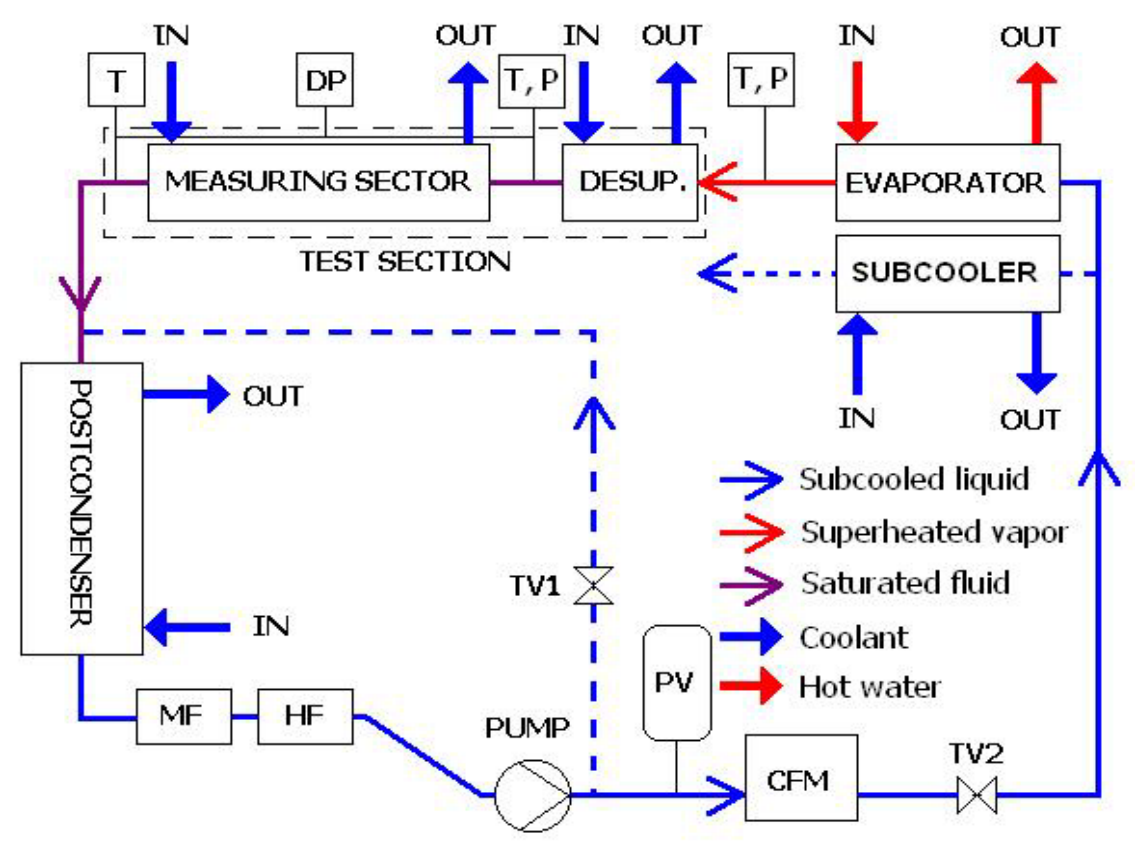

Figure 1: $\quad$ Scheme of the test rig.

The most important part of the test rig is the test section, which consists of two sectors. The first one, the pre-sector (PS) is used to prepare the desired inlet vapour quality, whereas the second one is actually the measuring sector (MS). Between the two sectors and downstream the MS, adiabatic stainless steel pipes are installed in order to reduce the axial heat conduction through the tube wall, to thermally detach the two sectors from the surrounding and to check the saturation state of the fluid by measuring the adiabatic wall temperature and the fluid pressure.

The test section is equipped with a number of thermocouples both in the wall and in the coolant channel along the sector; it is made from an $8 \mathrm{~mm}$ copper rod with a $0.96 \mathrm{~mm}$ internal bore - the minichannel.

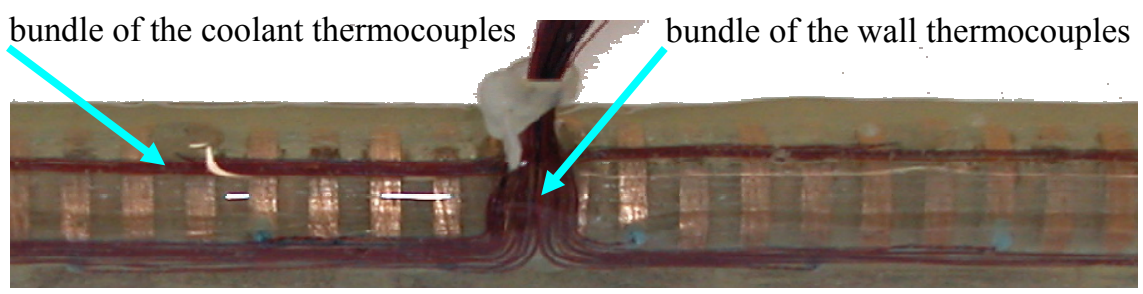

Figure 2: Close-up of the measuring sector during construction. 
A rather tortuous path for the secondary fluid is machined in the thick copper wall surrounded by an epoxy resin sheath (fig. 2) working as an insulator, armature and support for thermocouple wires. Crucial advantages of such a design reflect in the following characteristics:

- good coolant mixing and thus accurate temperature measurements along the measuring sector is essential to obtain a reliable water temperature profile, which is used for determination of local heat flux;

- both the continuous interrupting of the boundary layer, due to the rather complex coolant flow passage geometry, and the enhanced external wall surface area notably decrease the external heat transfer resistance, which is crucial for precise heat transfer coefficient measurements;

- thermocouple wires embedded in the copper wall are led outside the measuring sites through the epoxy resin without passing through the coolant flow: in this way the error of the temperature measurements due to axial heat conduction along the thermocouple wire and the spurious emf's build up for the presence of high temperature gradients is reduced to a minimum;

- The epoxy resin sheath does not only serve for accommodation of the thermocouple wires and for the insulation purpose; it also plays an important role as the test section's support.

\subsubsection{The test section length}

One of the first steps required for the test section design was the calculation of the test tube length. The coolant flow rate in the measuring sector that was initially built for the condensation studies was obtained from the calculated condensation heat flow rate and the desired overall coolant temperature rise in the sector. It was shown that, for a given test condition, any deviation of the tube length from the optimum one increases the experimental uncertainty of the test facility. Since the test tube was considered as a condenser, its size (length) depends severely on the external-side single-phase heat transfer. Good characteristics of the minichannel test tube require:

- High external heat transfer coefficient.

- Enhanced external surface area.

- Homogeneous wall surface temperature distribution along the channel.

- Low thermal resistance of the channel wall in radial direction.

- High thermal resistance of the channel wall in axial direction.

- Good coolant mixing.

- Low pressure drop.

The first two characteristics enhance the external heat transfer, which moves the leading thermal resistance toward the refrigerant side. In this way, the wall to refrigerant temperature difference is increased at given heat flow rate, while the relative error of the corresponding temperature difference measurement is decreased. Enhanced external heat transfer should be achieved so as to avoid systematic errors in wall temperature measurement due to local temperature variations. Furthermore, a high thermal conductivity of the tube wall decreases the associated temperature gradients and thus the wall temperature error due to deviation in temperature sensor positioning. On the other hand, the high thermal 


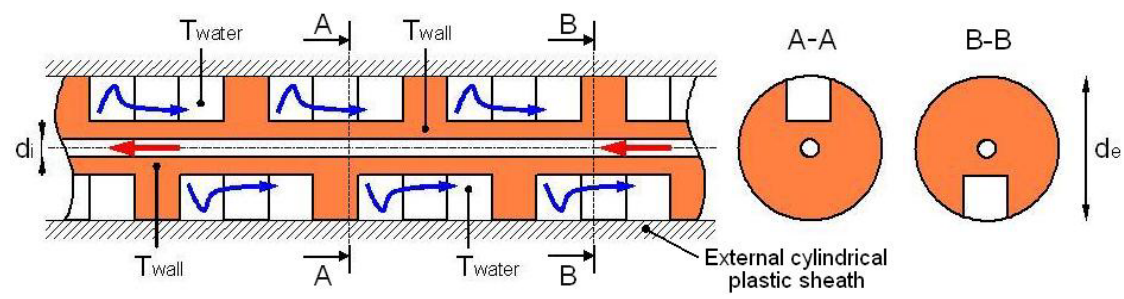

Figure 3: Schematic of the coolant flow passage geometry.

conductivity of the test tube also promotes the axial heat conduction. Even though, much smaller in comparison with the radial one, the axial heat conduction is additionally reduced by the multiple groves in the wall thickness of the present design. Additionally, a precise coolant temperature profile measurement is also of paramount importance for high performance heat transfer coefficient measurements. Insufficient coolant mixing is probably one of the most frequent reasons for systematic errors in fluid temperature measurement. On the other hand, a flow passage geometry that enables good fluid mixing and enhanced heat transfer are usually associated with significant pressure drop. In this context, the present coolant flow passage geometry has turned out to offer an excellent performance behaviour for high precision HTC measurements inside a single minichannel.

\subsubsection{Sensitivity of the temperature profile measurements}

Once the test section length and the test conditions were decided, positions for wall and coolant thermocouples had to be established. Optimal spacing between the temperature sensors depends on their nominal uncertainty and the temperature gradients along the test section. A minimum distance between two neighbouring thermocouples was defined by considering the axial displacement required for a minimum temperature rise exceeding the nominal error of the elements.

The minimum rational distance between two neighbouring thermocouples is defined according to the local temperature gradient along the measuring sector. In fact, a high heat transfer rate at the test section inlet results in high water and wall temperature gradients and thus a shorter distance between two thermocouples is needed to exceed the uncertainty of thermocouples. During condensation along the test tube, lower gas velocity induces lower heat transfer rate that requires less thermocouples to be set. The actual number of installed temperature sensors comes up as a compromise between a good temperature profile description and a reasonable design. Finally, there are 31 equally spaced thermocouples installed in the measuring sector: 16 in the wall and 15 in the water channel, both measuring reliable temperature profiles.

\subsubsection{Correction to the wall temperature measurement}

To estimate the correction of the wall temperature measurement due to the thermal resistance between the thermocouple's position and the inner wall surface, the temperature profiles around the central bore of the test tube have 
been plotted in fig. 4. When the thermocouples are positioned within a certain distance from the inner wall surface, a constant shifting of the measured wall temperature dependent on the heat flux is observed despite the variable external test conditions. For a thermocouple positioned $0.7 \mathrm{~mm}$ from the inner wall surface, temperature readings would result in around $0.23{ }^{\circ} \mathrm{C}$ lower values, for a given boundary condition, as compared to the internal wall surface temperature. Indeed, with respect to the acquired test conditions, appropriate correction for the wall temperature measurement is considered during data reduction.
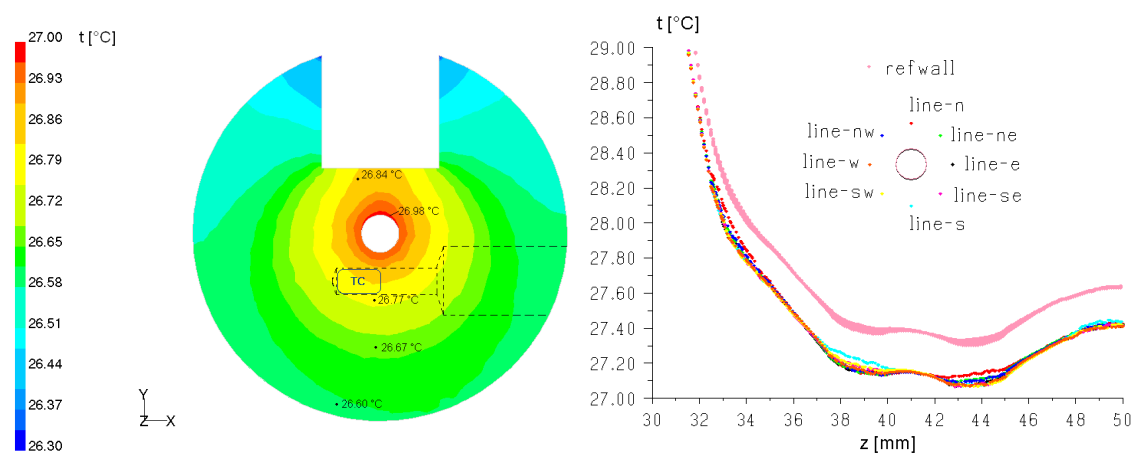

Figure 4: Wall surface temperature distribution.

\section{Experimental measurements}

\subsection{HTC during single phase flow, forced convective condensation, flow boiling and post dry-out}

Heat transfer coefficient measurements have been performed during single phase vapour and liquid flow, forced convective condensation, flow boiling and dryout (figs. 5, 6). Poor resistance to high temperatures of the epoxy resin $\left(<80{ }^{\circ} \mathrm{C}\right)$, maximum system pressure of the gear pump head $(<30$ bar $)$ and freezing point of the coolant - water $\left(0{ }^{\circ} \mathrm{C}\right)$ are the main system constraints. Schematics of the studied phenomena with R134a flowing within the single minichannel are shown in $\log \mathrm{p}$-h diagram of the figure 5 . Single phase heat transfer coefficient measurements during liquid and vapour flow have been performed for both cooling and heating modes. In single phase flow good agreement has been found of average experimental values versus the Gnielinski correlation [4, 5].

Besides, a precise measurement of the condensation heat transfer coefficients within the single minichannel has also been carried out (fig. 7 left). The present technique permits local measurements of the heat flux and saturation to wall temperature difference. This data is rare in literature since most authors measured condensation HTCs within multiport minichannels [6].

The averaged values of the heat transfer coefficient over the number of parallel minichannels do not give sufficient information on the phenomenon within a single minichannel. Besides, most of the experiments have been 


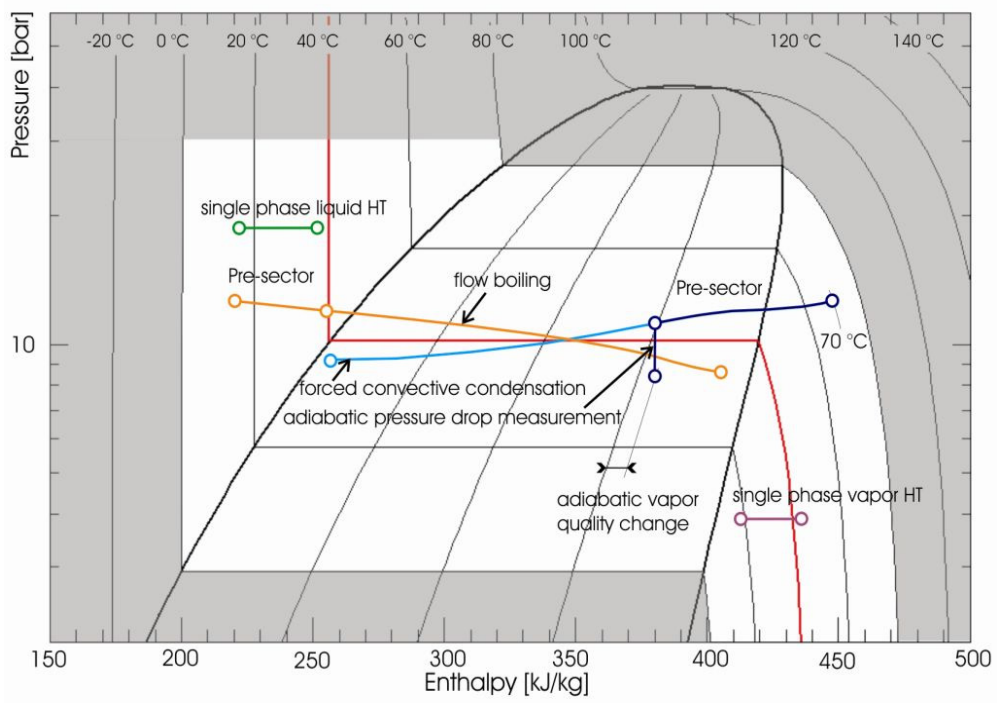

Figure 5: Different heat transfer coefficient and pressure drop measurements in $\log \mathrm{p}$-h diagram for R134a.

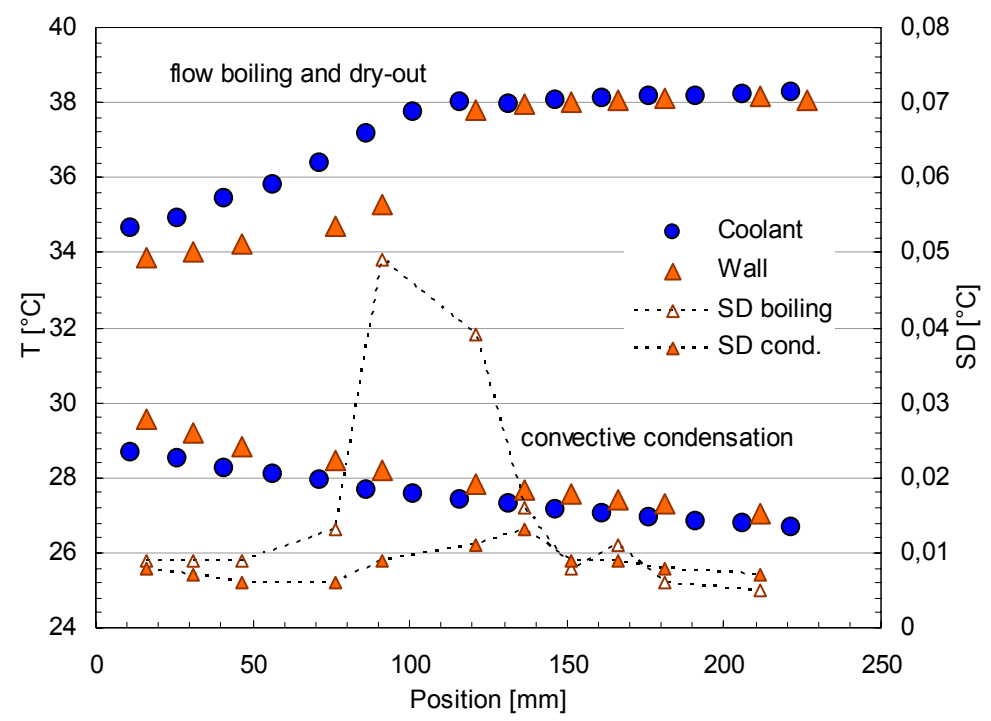

Figure 6: Temperature profiles for flow boiling and forced convective condensation HTC experiments with R134a at $\mathrm{G}=200 \mathrm{~kg} \mathrm{~m}^{-2} \mathrm{~s}^{-1}$.

performed with the Wilson plot technique, which does not require the wall temperature measurement for the heat transfer coefficient deduction, but calculates the coefficients from the variation of the external thermal resistance. 
This technique overpasses the difficulties raised by a reliable wall temperature measurement, but on the other hand, it presents a significant experimental error when the leading thermal resistance is on the coolant side.

Furthermore, local flow boiling heat transfer coefficients have also been measured with the present test apparatus (fig. 7 right). Literally the same measuring technique as the one adopted for condensation studies has been used $[7,8]$. In addition to temperature profiles, local temperature standard deviation values have turned out to be of crucial help for the definition of the dry-out region. There, during dry-out, the liquid film at the wall dries up with an oscillating process, leading to alternate change in thermal resistance. The region with rapid change in thermal resistance is thus denoted by a significantly higher wall temperature standard deviation as compared to the adjacent regions subject to boiling process or forced convection with gas (fig. 6).
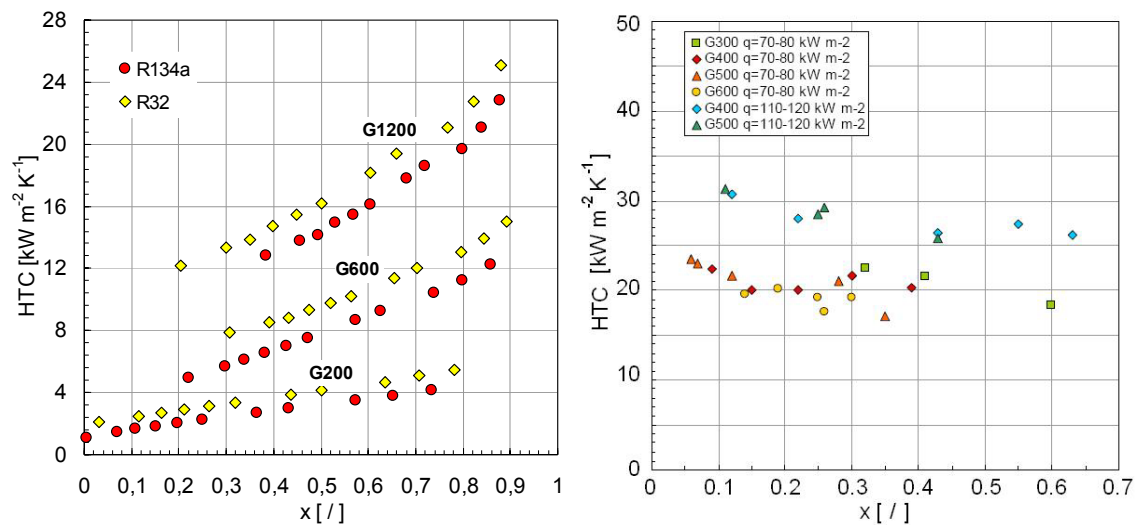

Figure 7: Experimental heat transfer coefficients versus vapour quality during condensation of R134a and R32 (left) and during flow boiling of R134a (right) inside a single minichannel.

\subsection{Pressure drop measurements during single phase flow, adiabatic and diabatic vapour-liquid flow}

Pressure drop experiments have been performed with two different fluids over a wide range of test conditions: during single-phase liquid and vapour flow (fig. 8 left), adiabatic two-phase flow (fig. 8 right) and during condensation flow. The experimental friction factor has been calculated from the overall measured pressure drop, the calculated values for two abrupt cross section area changes and the friction pressure drop within the adiabatic stainless steel capillaries [9], all included within the pressure measuring section. The experimental single phase friction factor is in good agreement with the Churchill [10] equation, which is reported over the entire span of Reynolds number. With regard to diabatic tests, pressure recovery due to condensation, boundary conditions for the two sudden geometry changes and mean value for the two phase multipliers 

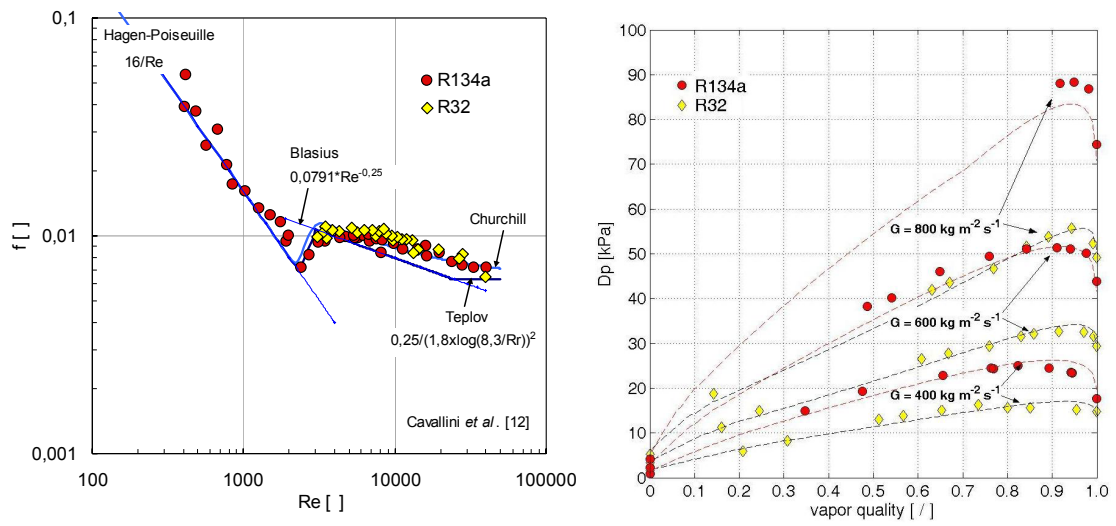

Figure 8: Experimental single phase friction factor versus Re (left) and overall two phase pressure drop vs. vapour quality (right).

have all been accounted for in the data reduction. The two-phase multiplier was calculated following Cavallini et al. [9] while the local pressure losses at the abrupt geometry changes were modelled according to Paliwoda [11].

\section{Conclusions}

The performance of the test apparatus for heat transfer and fluid flow studies within a single minichannel is described through the discussion of some design aspects and presentation of experimental test runs. This apparatus and the experimental technique for local HTC measurements with the use of a secondary cooling-heating medium have turned out to be highly efficient for experimental studies within a single minichannel.

\section{References}

[1] Kandlikar S.G. and Grande W.J., Evolution of microchannel flow passages - thermohydraulic performance and fabrication technology, Heat Transfer Engineering 24(1) (2003) 3-17.

[2] Kandlikar S. G., Garimella S., Li D., Colin S., King M. R., Heat transfer and fluid flow in minichannels and microchannels, Elsevier Ltd., ISBN: 00804-4527-6, 2006.

[3] Matkovic M., Experimental condensation inside minichannels, Ph.D. Thesis, Università di Padova, 2006, 209 p.

[4] VDI heat atlas / ed. Verein Deutscher Ingenieure, VDI-Gesellschaft Verfahrenstechnik und Chemieingenieurwesen (GVC), [Transl.: J. W. Fullarton]. - Düsseldorf: VDI-Verl., 1993.

[5] VDI-Wärmeatlas, Verein Deutscher Ingenieure, VDI-Gesellschaft Verfahrenstechnik und Chemieingenieurwesen (GVC), Springer-Verlag Berlin Hedelberg New York, 2002 
[6] Cavallini, A., Doretti, L., Matkovic, M. and Rossetto, L., Update on condensation heat transfer and pressure drop in minichannels, Heat Transfer Engineering 27(4) (2006a) 74-87.

[7] Cavallini A., Bortolin S., Del Col D., Matkovic M., Rossetto L., Local Heat Transfer Coefficient During Flow Boiling inside Circular Minichannel, ASME-JSME Thermal Engineering Summer Heat Transfer Conference, July 8-12, 2007, Vancouver, British Columbia, Canada.

[8] Del Col D., Cavallini A., Bortolin S., Matkovic M., Rossetto L., Heat Transfer Coefficient during Flow Boiling of R134a in a Circular Minichannel, $5^{\text {th }}$ European Thermal-Sciences Conference, 18-22 May, 2008, Eindhoven, Netherlands.

[9] Cavallini A., Del Col D., Matkovic M., Rossetto L., Frictional Pressure Drops During Vapour-Liquid Flow in Minichannels: Experimental Data and Modelling, Micro/Nanoscale Heat Transfer International Conference, Tainan, Taiwan, 2008, MNHT2008-52282.

[10] Churchill, S.W., "Friction factor equation spans all fluid-flow regimes", Chemical Engineering 45, 1977, pp. 91-92.

[11] Paliwoda, A., "Generalized method of pressure drop calculation across pipe components containing two-phase flow of refrigerants", Rev. Int. Froid, 15(2), 1992, pp. 119-125.

[12] Cavallini A., Del Col D., Matkovic M., Rossetto L., Pressure Drop During Two-Phase Flow of R134a And R32 in a Single Minichannel, Submitted to Journal of Heat Transfer, 2008. 